\title{
Altered Sexual Partner Preference in Male Ferrets Given Excitotoxic Lesions of the Preoptic Area/Anterior Hypothalamus
}

\author{
R. G. Paredes ${ }^{\mathrm{a}}$ and M. J. Baum \\ Department of Biology, Boston University, Boston, Massachusetts 02215
}

Numerous experiments suggest that perinatal exposure of male vertebrates to testosterone $(T)$, or its estrogenic metabolites, masculinizes aspects of coital function, including males' characteristic preference to seek out and mate with a female as opposed to another male conspecific. Other research has shown that this perinatal action of sex steroids also masculinizes aspects of neuronal morphology in the medial preoptic area/anterior hypothalamus (mPOA) AH). We asked whether neurons of the mPOAAH contribute to males' preference to mate with a female. The ferret is an ideal species in which to ask this question. When tested in a T-maze after gonadectomy and treatment with estradiol benzoate (EB), female ferrets prefer to approach and receive neck grips from a stimulus male whereas males prefer to approach and neck grip an estrous female. In the minority of trials when EB-treated males approach a stimulus male, they occasionally receive a neck grip to which they display receptive postures as opposed to agonistic behaviors. In Experiment 1 castrated, EB-treated male ferrets which received bilateral infusions of the NMDA excitotoxin, quinolinic acid aimed at the dorsomedial POA AH, preferred to approach a stimulus male significantly more often than groups of control males which either received a sham lesion, received a unilateral mPOAAH lesion or in which bilateral infusions of quinolinic acid produced no histologically detectible excitotoxic damage to the mPOAAH. Males with bilateral mPOA/AH lesions also displayed neck gripping on a significantly lower percentage of trials than control males when they approached the stimulus female. Ovariectomized, EB-treated female ferrets with bilateral mPOAAH lesions, like control females, preferred to approach and receive neck grips from a stimulus male. The males used in Experiment 1 had never experienced circulating levels of $\mathrm{T}$ characteristic of the breeding season. Therefore, in Experiment 2 prepubertally gonadectomized males and females were treated chronically with a high dose of $\mathrm{T}$ propionate (TP) and tested several times with a receptive female prior to brain surgery. Males which received bilateral excitotoxic lesions of the mPOA/AH neck

\footnotetext{
Received Mar. 21, 1995; revised May 30, 1995; accepted June 6, 1995.

This research was supported by NIH Grant HD21094 and by NIMH Re search Scientist Award MH00392 to M.J.B. We thank Elgantina Kica for ex cellent technical assistance and the Boston University Laboratory Animal Care Facility staff for their devoted attention of our ferret colony.

Correspondence should be addressed to Dr. Michael Baum, Department of Biology, Boston University, 5 Cummington Street, Boston, MA 02215.

Present address: Department of Psychology, Anahuac University, Box 10 844, Mexico, D.F. 11000.

Copyright (C) 1995 Society for Neuroscience $0270-6474 / 95 / 156619-12 \$ 05.00 / 0$
}

gripped and mounted stimulus females significantly less than control males. Again, when given EB followed by T-maze tests of partner preference, males with bilateral mPOA/AH lesions, like sham-operated female controls, preferred to approach the sexually active stimulus male significantly more often than control males, which preferred to approach the stimulus female. Our results suggest that neurons in the MPOA/AH play an important role in the integration of sensory cues which determine heterosexual partner preference in the male ferret, in addition to facilitating masculine coital performance.

[Key words: ferret, partner preference, medial preoptic area/anterior hypothalamus (mPOA/AH), excitotoxic lesions, sex behavior, sex steroids]

Studies using several vertebrate species (birds, Adkins-Regan and Ascenzi, 1987; rat, Bakker et al., 1993; ferret, Baum et al., 1990b; pig, Ford, 1983) suggest that males prefer to approach and copulate with female, as opposed to other male, conspecifics because of the perinatal actions of testosterone $(T)$, or estrogenic metabolites of $\mathrm{T}$. The male ferret possesses a nucleus in the dorsomedial preoptic area/anterior hypothalamus (mPOA/AH), called the male nucleus of the POA/AH (Mn-POA/AH), which is absent in females (Tobet et al., 1986a). A homologous structure has been identified in male rats (Gorski et al., 1978), gerbils (Commins and Yahr, 1984) and in several other species including man (reviewed in Tobet and Fox, 1992). In rat and ferret the sexually dimorphic features of mPOA/AH morphology present in males result from the perinatal action of estrogen, formed in the brain from circulating $\mathrm{T}$ (Dohler et al., 1984; Tobet et al., 1986b). These observations raise the question of whether males' heterosexual partner preference is influenced by sexually dimorphic neurons of the mPOA/AH? Numerous studies (see Discussion for references) have shown that lesions of the $\mathrm{MPOA} /$ AH disrupt masculine coital function; however, to our knowledge nobody has studied the effect of such lesions on males' preference to seek out and mate with a female as opposed to a male partner. We conducted two such experiments using ferrets which had sustained bilateral, axon-sparing excitotoxic lesions of the mPOA/AH in response to infusions of the NMDA receptor agonist, quinolinic acid. In a previous study (Stockman et al., 1985) we found that following gonadectomy and treatment with a high dose of estradiol benzoate (EB), male ferrets preferred to approach an estrous temale as opposed to a stud male in a T-maze. These males typically performed neck grips on stimulus females after choosing to approach them, probably because estrogen, formed via neural aromatization of $T$, normally contributes to the activation of masculine sexual behavior in this species (Carroll et al., 1988). In the minority of tests when EB- 
treated males chose to approach the stud male, they often received neck grips to which they displayed a female-like receptive posture. Estrogen-treated female ferrets preferred to approach and bc ncck gripped by thc stimulus male. Fighting was rarely seen in these tests of preference, leading us to infer that the primary incentive to approach either stimulus in our testing situation was the opportunity to engage in sexual as opposed to agonistic behavior.

In Experiment 1 we studied the effects of bilateral excitotoxic mPOA/AH lesions on partner preference and masculine sexual behavior of gonadectomized, EB-treated male and feniale ferrets. Males with bilateral $\mathrm{mPOA} / \mathrm{AH}$ lesions preferred to approach a stud male, as opposed to an estrous female, significantly more often than control males. However, the magnitude of the preference for an estrous female seen in sham-operated control males was considerably smaller than that seen in previous studies (Stockman et al., 1985; Baum et al., 1990b), perhaps because the ferrets used in this study had been gonadectomized and first given EB prior to puberty. As a result, these males had never mated with females while $\mathrm{T}$ was circulating. Therefore, in a second Experiment all subjects were gonadectomized, treated daily with a high dose of $\mathrm{T}$ propionate (TP), and given experience with an estrous female prior to being tested for coital behavior. Excitotoxic mPOA/AH lesions or sham-operations were then administered, and masculine coital behavior was again assessed while subjects continued to receive TP. Subjects were then switched to daily injections of a high dose of EB prior to receiving T-maze tests of partner preference. Again, males with hilateral lesions of the $\mathrm{mPOA} / \mathrm{AH}$ preferred to approach the stimulus male on significantly more trials than control males.

\section{Materials and Methods}

\section{Animals}

Most of the ferrets used in these studies were purchased from Marshall Research Farms (North Rose, NY) when they were approximately 6 months of age and had not yet entered their first breeding season. Additional animals which had been born in our colony were also used when they were 6 months of age. All ferrets were housed individually in modified rabbit cages and were fed Purina ferret chow each morning. Water was always available. Colony lights were on each day between 0600 and 2200 hours. All animals were gonadectomized under sterile conditions using ketamine $(35 \mathrm{mg} / \mathrm{kg})$ and xylazine $(4 \mathrm{mg} / \mathrm{kg})$ anesthesia. Females were also hysterectomized at the time their ovaries were removed. Stimulus females were injected daily with EB $(15 \mu \mathrm{g} / \mathrm{kg})$. Stimulus (stud) males were injected daily with TP $(5 \mathrm{mg} / \mathrm{kg})$.

\section{Brain surgery}

Brain lesions were performed under sterile conditions using pentobarbital $(35 \mathrm{mg} / \mathrm{kg})$ anesthesia and a Kopf stereotaxic instrument equipped with a squirrel monkey head adaptor and ferret ear bars. The NMDA receptor agonist, Quinolinic acid $(0.12 \mathrm{M}$ in PBS, pH 7.4; Sigma, St. Louis, MO), was freshly prepared $0.5-3 \mathrm{hr}$ prior to use and kept in darkness on ice. Stereotaxic coordinates for the dorsomedial POA/AH were determined empirically to be 4.5 or $4.3 \mathrm{~mm}$ anterior to the interaural line, $0.4 \mathrm{~mm}$ lateral to the sub-dural midline sinus, and 12.0 or $11.9 \mathrm{~mm}$ below the dura in males and females, respectively. We attempted to place large excitotoxic lesions bilaterally (Experiments 1 and
2) by lowering a $1 \mu \mathrm{l}$ Hamilton syringe (26 gauge, blunt tip) into the brain and injecting $0.75 \mu \mathrm{l}$ of quinolinic acid. In a subgroup $(n=8)$ of males used in Experiment 2 we attempted to combine damage specific to the Mn-POA/AH on the left side with a large mPOA/AH lesion on the right side. These males received $0.25 \mu \mathrm{l}$ and $0.75 \mu \mathrm{l}$ of quinolinic acid into the left an right sides of the MPOA/AH, respectively, via a 28 gauge needle (blunt tip) attached with PE tubing to a $1 \mu$ l Hamilton syringe. In a previous study (Cherry and Baum, 1990) we had successfully used similar assymetric electrolytic lesions to assess the function of the Mn-POA/AH in male ferrets. All infusions of excitotoxin were given slowly over $5 \mathrm{~min}$, and the injection needle was left in place for an additional $5 \mathrm{~min}$ in order to minimize the leakage of quinolinic acid back along the needle path.

\section{Behavioral testing}

Coital behaviors. While maintaining a neck grip, the male mounts the female and exhibits episodes of pelvic thrusting which after $30 \mathrm{~min}$ or more lead to a single, prolonged intromission (without thrusting) which may last for more than 1 hr (Baum, 1976). In response to a male's neck grip, a receptive female will exhibit a limp, unresisting posture whereas a nonreceptive female will actively resist (by biting or fleeing) any neck grip attempt by a stud male. Tests of masculine sexual behavior were conducted by placing a subject together with an estrous stimulus female in a test cage with a Plexiglas front and a grid floor. An observer used a laptop computer to record the duration of neck gripping, mounting, and pelvic thrusting displayed by subjects during two 20 min tests, given on separate days. Typically, intromission and ejaculation were not seen tests of this length.

T-Maze tests of partner preference. Male and female subjects were tested for their preference to approach and interact with either a stud male or a sexually receptive estrous female (Johnson and Tiefer, 1972; Stockman et al., 1985; Baum et al., 1990b). The two stimulus animals were always placed in the same, respective goal boxes, located $2.3 \mathrm{~m}$ from the start compartment of the T-maze. These goal boxes had wiremesh doors and were located at the ends of the two lateral arms of the maze. The walls of the lateral arm leading to the stimulus male were covered with horizontal black and white stripes. The rest of the maze was painted a dull black. Thus, subjects had consistent visual and spatial cues associated with an approach to each type of stimulus animal. In a previous study (Stockman et al., 1985) we counterbalanced the location of the stimulus male and female in our T-maze assessment of ferrets' sexual preference. We subsequently became concerned that this procedure might inadvertently have led to a cross-contamination of the goal boxes by chemosensory cues from the respective stimulus animals. Therefore, in more recent studies we have kept the two types of stimulus ferret in the same respective goal boxes during all tests. Thus, in Baum et al. (1990b) subjects were required to make a right turn to approach the stimulus male and a left turn to approach the stimulus female whereas in the present studies subjects were required to make a left turn to approach the male and a right turn to approach the female. In all of these experiments, gonadectomized, EB-treated, control males preferred to approach the stimulus female whereas EB-treated, control females preferred the stud male. The consistency of this finding over several experiments, regardless of the postion (left or right) of the stimulus animals in a particular study, rules out the possibility that the present results are confounded by a trivial difference in the preference of male and female subjects to turn to the left or right when approaching the goal boxes of the T-maze.

Subjects received 8 "free" and 8 "guided" trials on each of 3 separate test days, making a total of 24 free and 24 guided trials. During free trials the subject was placed in the start box, and after $30 \mathrm{sec}$ the door was raised and the subject was allowed to run the maze. After the subject had moved into one of the two lateral compartments and approached a particular goal box, its screen door was opened and the

Figure 1. Photomicrographs of the dorsomedial preoptic area/anterior hypothalamus (mPOA/AH) of representative sham-operated male $(A, C)$ and female $(E, G)$ ferrets, and of a male $(B, D)$ and a female $(F, H)$ in which excitotoxic damage, including neuronal depletion and gliosis, is evident as a result of earlier infusion of the NMDA agonist, quinolinic acid. The nondimorphic ventral nucleus of the mPOA/AH (surrounded hy black arrowheads in $A$ and $E$ ) was damaged by excitotoxin treatments in both the lesioned male $(B)$ and female $(F)$. The sexually dimorphic male nucleus of the POA/AH in the male (surrounded by open arrowheads in $A$ ) and the equivalent region in the female were also damaged by excitotoxin treatments. For each subject, the black arrow in the low magnification photomicrograph points to the location within the dorsomedial PO $\Lambda / \mathrm{H}$ which is shown in the corresponding high magnification photomicrograph. $3 v$, third ventricle. Scale bars: $A, 200 \mu \mathrm{m} ; C, 10 \mu \mathrm{m}$. 

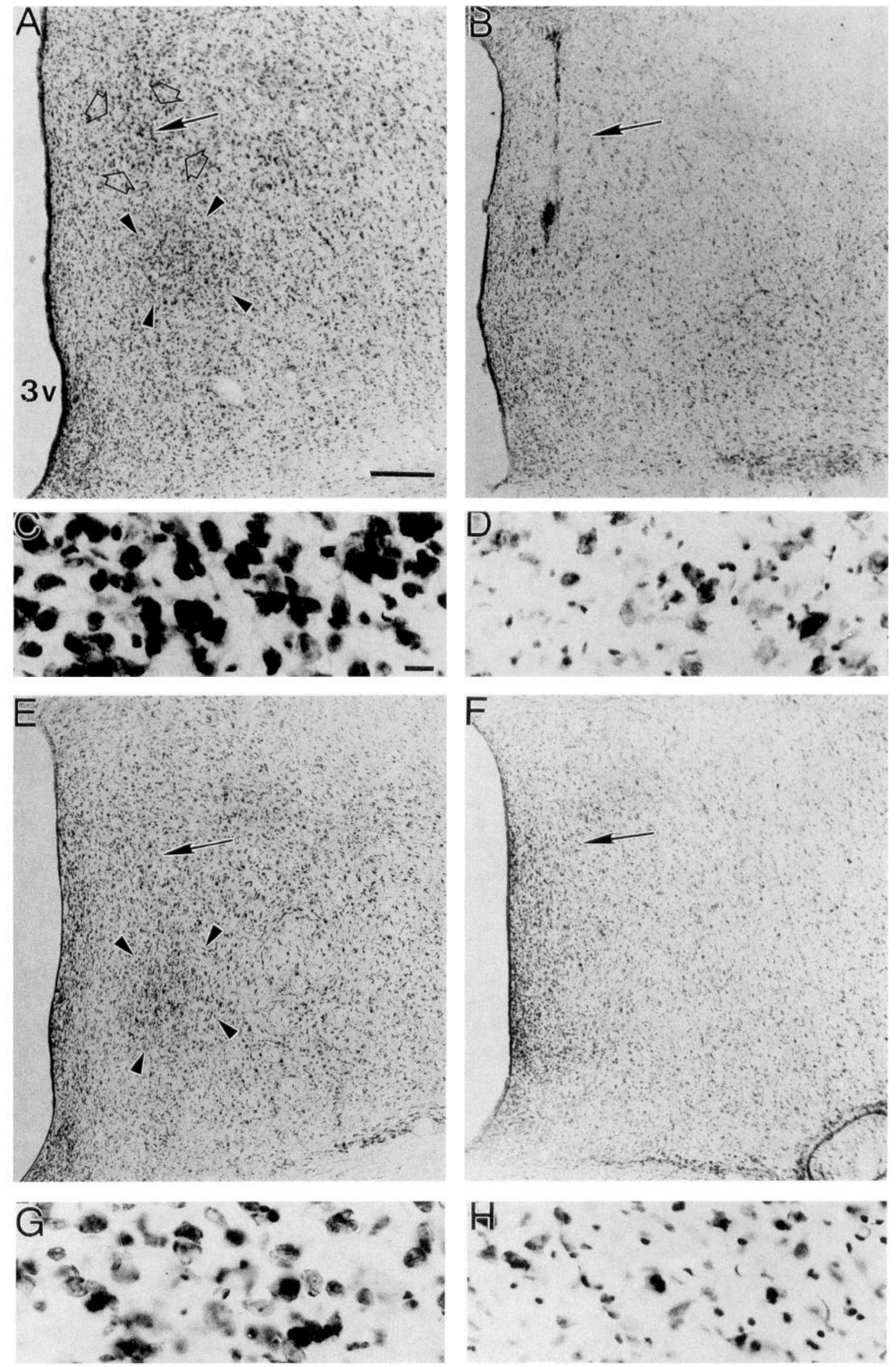
stimulus animal and the subject were allowed to interact for $1 \mathrm{~min}$. The stimulus chosen and the approach latency were recorded, along with details (see below) of any sexual or agonistic interaction between the subject and a stimulus ferret. The animals were then separated and the subject was returned to the start box whereupon a guided trial was run. Guided trials were identical to free trials, except that the door to the previously chosen lateral compartment was closed. Thus, the subject was encouraged to approach the stimulus animal not chosen on the previous free trial. In this way we intended to remind the subject being tested of the alternative stimulus animal which was also available during the next free trial. Interspersion of these guided trials among free trials also insured that on each test day each subject was exposed during an equal number of trials to both stimulus animals. During any trial subjects could choose not to leave the start compartment or approach one of the goal boxes, thereby avoiding any interaction with a particular stimulus. A particular trial was terminated if the subject failed to approach one of the goal boxes within $120 \mathrm{sec}$. This almost never occurred. In order to acquaint them with the identity and location of the stimulus ferrets, all subjects initially received 16 guided trials alternating between an estrous female and a stud male on a day prior to the actual test sessions. Subjects' partner preference was indexed as the percentage of the 24 free trials (given over 3 test days) during which either the stimulus male or female was approached. A further indication of subjects' motivation to approach either stimulus was reflected in the mean approach latencies to these stimuli, during either free or guided trials. A record was kept of the percentage of trials over 3 test days (free and guided trials, combined) during which the stud male neck gripped a subject after being approached (index of subjects' sexual attractivity; Beach, 1976) and the percentage of trials during which a neck grip by the stud led to a receptive response by the subject (index of subjects' sexual receptivity). Also, a record was kept of the percentage of trials during which subjects neck gripped the estrous female, following an approach to this stimulus (an index of subjects' masculine coital behavior). Finally, a record was kept of the percentage of trials with fighting between subjects and either stimulus.

\section{Sequence of hormone treatments and tests}

Experiment 1. In conjunction with another study (to be reported elsewhere), gonadectomized ferrets of both sexes received daily subcutaneous injections of increasing weekly doses of EB $(0,8$, and $15 \mu \mathrm{g} / \mathrm{kg})$ and were tested over a 3 week period in the T-maze for their preference to approach and interact with either a stud male or a sexually inactive, castrate male. Subjects' masculine coital behavior was also tested while they received the $15 \mu \mathrm{g} / \mathrm{kg}$ dose of $\mathrm{EB}$. Hormone treatments were stopped, and subjects then received bilateral infusions of either quinolinic acid or PBS vehicle aimed at the dorsomedial POA/AH. Two weeks after surgery animals were given the same sequence of $E B$ treatments and preference tests as they had received prior to brain surgery, followed by tests of masculine coital behavior. Then, while they continued to receive daily subcutaneous injections of $15 \mu \mathrm{g} / \mathrm{kg} \mathrm{EB}$, all subjects were given $T$-maze tests of preference for a stud male versus an estrous female.

Experiment 2. Gonadectomized subjects were treated daily for at least $10 \mathrm{~d}$ with a dose of TP $(5 \mathrm{mg} / \mathrm{kg})$ which optimally activates masculine sexual behavior in castrated male ferrets (Lambert and Baum, 1991). While receiving TP, subjects were placed with an estrous female for 1 $\mathrm{hr}$; behavior was not scored during this session. Subjects were tested on two subsequent days with an estrous female for masculine coital behavior. Subjects then received bilateral mPOA/AH infusions of either quinolinic acid or PBS vehicle, while continuing to receive daily injections of TP. One week later all animals were tested again for masculine coital behavior. Treatment with TP was stopped, whereupon all subjects were injected for at least $15 \mathrm{~d}$ with EB $(15 \mu \mathrm{g} / \mathrm{kg} / \mathrm{d})$ prior to receiving T-maze tests of partner preference.

\section{Histology}

After the completion of behavioral tests, all subjects were deeply anesthetized using pentobarbitol $(35 \mathrm{mg} / \mathrm{kg}$ ) and were perfused via the heart with $0.9 \%$ saline followed by $10 \%$ formalin. Brains were removed and placed in $10 \%$ formalin $/ 30 \%$ sucrose for $36 \mathrm{hr}$. A sledge microtome was used to cut $40 \mu \mathrm{m}$ thick frozen coronal sections which were mounted on gelatin-coated slides and were stained with cresyl violet. The presence in the MPOA/AH of neuronal depletion and gliosis was used to determine the boundaries of excitotoxic damage in subjects which had received infusions of quinolinic acid. Without knowledge of subjects' behavioral data, one investigator drew the extent of lesion damage for each subject using a template series of drawings made in the coronal plane at $240 \mu \mathrm{m}$ intervals through the ferret forebrain. A second investigator then confirmed the boundaries of the lesion damage drawn for each subject. Any disagreements about these boundaries were resolved, whereupon subjects were assigned to different treatment groups, and their behavioral data were summarized. The serial drawings of the boundaries of excitotoxic damage from individual subjects were later superimposed in order to create summary drawings which portrayed the maximal extent and any overlap in excitotoxic damage for all members of each group of ferrets in both experiments.

\section{Statistical analysis}

Within each experiment, data summarized as the percentage of trials during which a particular behavior occurred were first analyzed across all groups using a one-way Kruskal-Wallis analysis of variance by ranks. When a significant $(p<0.05) H$ value was obtained, post-hoc comparisons of pairs of group means were made using two-tailed MannWhitney $U$ tests. In Experiment 2 data from tests of coital behavior were analyzed using a two-way ANOVA, with subsequent post-hoc comparisons of group means being made using Newman-Keuls tests. Binomial tests were used to determine whether the proportion of subjects in particular treatment groups (animals from Experiments 1 and 2 combined) which preferred to approach either the male or female stimulus in $>50 \%$ of free trials differed from what would be expected by chance.

\section{Results}

\section{Extent of excitotoxic lesion damage}

Examples of the neuronal depletion, coupled with extensive gliosis, which occurred in the dorsomedial POA/AH of male and female ferrets after infusion of quinolinic acid, are shown in Figure 1. Schematic diagrams of the maximal extent as well as the common overlap of excitotoxic lesions given to ferrets in Experiment 1 are shown in Figure 2. Bilateral lesions in both males (Fig. 2B) and females (Fig. 2C) were centered in the dorsomedial $\mathrm{POA} / \mathrm{AH}$, so as to damage the Mn-POA/AH bilaterally in males. In both sexes, however, bilateral lesions invariably extended to include more lateral and ventral portions of the POA/AH. In some male and female subjects only unilateral excitotoxic damage was found (a summary of unilateral lesion damage in males from Experiment 1 is shown in Fig. $2 A$ ) even though quinolinic acid had been infused bilaterally into these animals. In addition, groups of male and female ferrets were identified in which no excitotoxic damage to the forebrain could be found, even though these ferrets had also received bilateral infusions of quinolinic acid. We cannot rule out the possibility that the absence of excitotoxic damage after infusion of guinolinic acid into the dorsomedial POA/AH of particular animals resulted from a leakage of the neurotoxin into the cerebral ventricles. It is important to note, however, that considerable variation among different species has been reported in the consistency of excitotoxic damage after the infusion of NMDA agonists into the mPOA/AH. Thus in marmosets (Dixson and Hastings, 1992) and hamsters (M. Hastings, personal communication), as in ferrets (present studies), infusions of NMDA agonists often failed to elicit an excitotoxic response in the POA/AH. By contrast, in rats (Baum and Everitt, 1992) and gerbils (Yahr and Gregory, 1993) more consistent excitotoxic effects of NMDA receptor agonists have been obtained following mPOA/AH infusions. The explanation for this variability in some species, and for the species differences in POA/AH responsiveness to NMDA agonists is unknown. In the present experiments we used positive histological criteria (neuronal depletion, coupled with gliosis) to assign individual ferrets to groups prior to analysis of the 


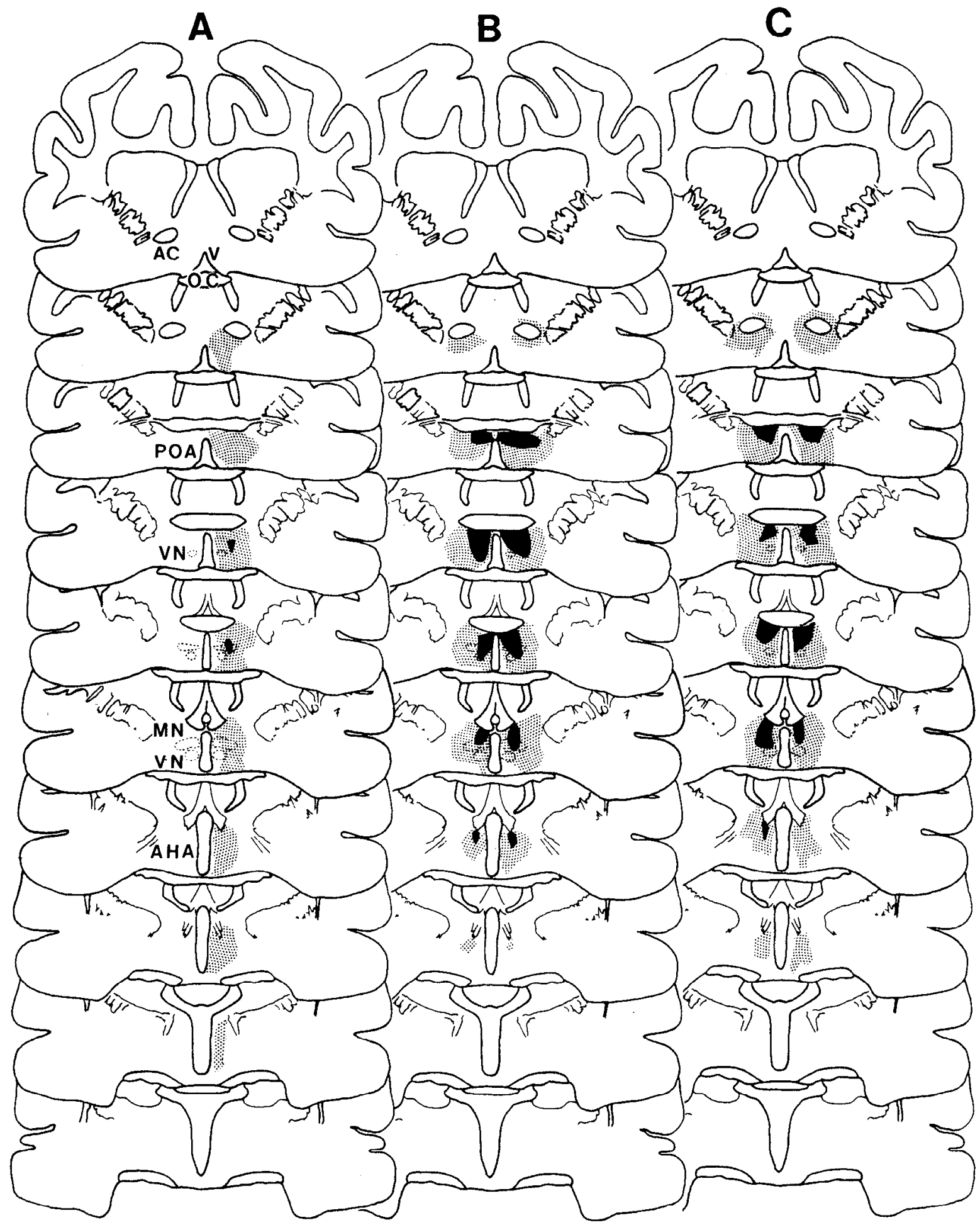

Figure 2. Composite diagrams of the extent of excitotoxic damage caused by infusions of quinolinic acid into the dorsomedial preoptic area/ anterior hypothalamus (mPOA/AH) of groups of ferrets from Experiment 1. Hatched regions depict the maximal extent of tissue which sustained damage in any member of a particular group; black regions depict overlap of excitotoxic damage for all members of a particular group. Data are shown for six males with unilateral excitotoxic damage (two and four males with damage in the left and right sides, respectively-sumnarized here on one side for ease of presentation) $(A)$ and for four males $(B)$ and six females $(C)$ with bilateral excitotoxic damage to the $\mathrm{mPOA} / \mathrm{AH}$. Abbreviations: $P O A$, preoptic area; $v n$, non-dimorphic, ventral nucleus of the mPOA/AH; $m n$, sexually dimorphic male nucleus of the mPOA/AH;
$A H A$, anterior hypothalamic area. 
behavioral results. Neuronal depletion and gliosis were never observed in sham-operated ferrets which received infusions of PBS into the MPOA/AH.

The localization of mPOA/AH damage (both bilateral and unilateral) obtained in Experiment 2 after bilateral infusions of quinolinic acid (Fig. 3) closely resembled that obtained in Experiment 1 , even in the subgroup of eight males in which the volume of quinolinic acid infused into the left mPOA/AH was reduced from 0.75 to $0.25 \mu \mathrm{l}$. Again, groups of males were identified with either unilateral or no detectible excitotoxic $\mathrm{mPOA} / \mathrm{AH}$ damage despite having received bilateral infusions of quinolinic acid into the mPOA/AH. In both experiments the behavioral data from these groups closely resembled those of sham-operated subjects which received PBS vehicle. The behavioral data from these different control groups contrasted dramatically with results from males bearing bilateral excitotoxic lesions of the mPOA/AH (see below).

\section{Behavioral results}

Experiment 1. Male subjects with bilateral $\mathrm{mPOA} / \mathrm{AH}$ lesions showed a clear preference to approach the stud male instead of the receptive female ( $H=15.1$, df $=7, p<0.05$; Fig. 4). By contrast, sham-operated males showed an equal preference for the two types of stimulus animal whereas males with unilateral excitotoxic lesions or no detectible excitotoxic damage to the mPOA/AH preferred to approach a receptive female. Females bearing bilateral lesions of the $\mathrm{mPOA} / \mathrm{AH}$, like sham-operated females, females with unilateral mPOA/AH damage and females with no detectible excitoxic damage, consistently preferred to approach the stud male. There were no significant effects of bilateral mPOA/AH lesions on approach latencies to either stimulus within groups of male and female subjects. Mean approach latencies in free trials to either stimulus ranged from 6-13 sec. for groups of males and from 4-11 sec. for groups of females. When male subjects approached the stud male, they received neck grips in an equivalent low percentage of trials, regardless of treatment group (Fig. 5, top panel). By contrast, females in all four treatment groups nearly always received neck grips on trials when they approached a stud male $(H=36.4$, df $=7, p$ $<0.01)$. All groups of males and females were highly receptive in response to neck grips by the stud male (Fig. 5, bottom panel). On trials when they approached a sexually receptive stimulus female, males with bilateral lesions of the $\mathrm{mPOA} / \mathrm{AH}$ were significantly less likely than males in the three other groups to display neck grip behavior $(H=28.9$, df $=7, p<0.01)$; females in all four groups displayed equivalent, low levels of this behavior (Fig. 6, top panel). Compared with their response to an estrous female, subjects displayed low levels of neck grip behavior when they approached a stud male (Fig. 6, bottom panel), although in this case all groups of male subjects neck gripped the stimulus male significantly more often than females $(H=35.2, \mathrm{df}=7, p<0.01)$. No fighting was observed in this experiment. In tests with estrous females there were no significant effects of bilateral lesions of the MPOA/AH on males' coital behavior (data not shown), perhaps because the EB treatment used in this experiment activated only moderate levels of this behavior. Thus, in preoperative tests of coital behavior shamoperated, EB-treated males (Experiment 1) mounted the estrous stimulus female for $209 \pm 132 \mathrm{sec}$ whereas in Experiment 2 (below) sham-operated, TP-treated males displayed mounting for $724 \pm 109 \mathrm{sec}$.

Experiment 2. As in Experiment 1, males bearing bilateral lesions of the mPOA/AH were significantly more likely to approach the stud male than were males bearing sham lesions, unilateral excitotoxic lesions or nondetectible damage to the $\mathrm{mPOA} / \mathrm{AH}$, all of which preferred to approach the estrous female ( $H=17.5$, df $=4, p<0.01$; Fig. 7). Sham-operated females clearly preferred to approach the stud male. As in Experiment 1 , there were no significant group differences in latencies to approach the stimulus male or female in either free or guided trials (mean approach latencies in free trials to either stimulus ranged from 7-13 sec. in the five groups). When they approached the stud male, all four groups of male subjects received a neck grip on an equivalent, low percentage of trials, compared with female controls which received neck grips on nearly $100 \%$ of the trials; all groups displayed equivalent, high levels of receptivity when neck gripped by the stud male (data not shown). As in Experiment 1, males with bilateral mPOA/AH lesions neck gripped the stimulus female on a significantly lower percentage of trials than other groups of males (data not shown). Occasional fights occurred between male subjects and stud males; however, there were no group differences in the incidence of this behavior (data not shown). When tested preoperatively while receiving a high dosage of TP, males in all four groups displayed high levels of neck grip and mounting behavior (Fig. 8). When tested postoperatively while still receiving $\mathrm{TP}$, males with bilateral $\mathrm{mPOA} /$ $\mathrm{AH}$ lesions showed significant reductions in these behaviors. Female subjects displayed very low levels of masculine sexual behavior before and after sham surgery (neck grip: $F_{4,79}=35.8$, $p<0.01$; mount: $F_{4,79}=26.2, p<0.01$ ).

When partner preference data for particular treatment groups were combined from Experiments 1 and 2, the following outcome was obtained: of control males (i.e., males given sham lesions, unilateral mPOA/AH lesions, or in which no brain lesion could be detected even after central infusions of quinolinic acid) $64 \%(25 / 39)$ preferred to approach the stimulus female on $>50 \%$ of free trials $(p<0.05$, one-tailed binomial test). By contrast, $88 \%$ (23/26) of control females preferred to approach the stimulus male on $>50 \%$ of the free trials $(p<0.01$, onetailed binomial test). Finally, $81 \%$ (13/16) of males with bilateral lesions of the mPOA/AH preferred to approach a stimulus male on $>50 \%$ of free trials ( $p<0.02$, two-tailed binomial test).

\section{Discussion}

The major finding of both experiments was that gonadectomized, EB-treated male ferrets bearing bilateral excitotoxic lesions centered in the dorsomedial POA/AH preferred to approach and interact with a sexually active male conspecific, in contrast to control males which generally preferred to approach an estrous female. In Experiment 1 the preference of males with bilateral $\mathrm{mPOA} / \mathrm{AH}$ lesions for the stimulus male was at least as great as that of the four groups of females tested, perhaps because these males had never been exposed to estrous females while high circulating titers of $\mathrm{T}$ were present. In Experiment 2 castrated males all received $\mathrm{T}$ and sexual experience prior to brain surgery. Again, when treated with EB males with bilateral mPOA/ $\mathrm{AH}$ lesions preferred to approach the stimulus male significantly more often than control males, although the magnitude of the preference was lower than that of female controls. These results suggest that the sexual dimorphism in ferrets' sexual partner preference, seen in gonadectomized, EB-treated control groups tested in this study and in past experiments (Stockman et al., 1985; Baum et al., 1990b), depends on the functional integrity of neurons in the male's dorsomedial POA/AH. 


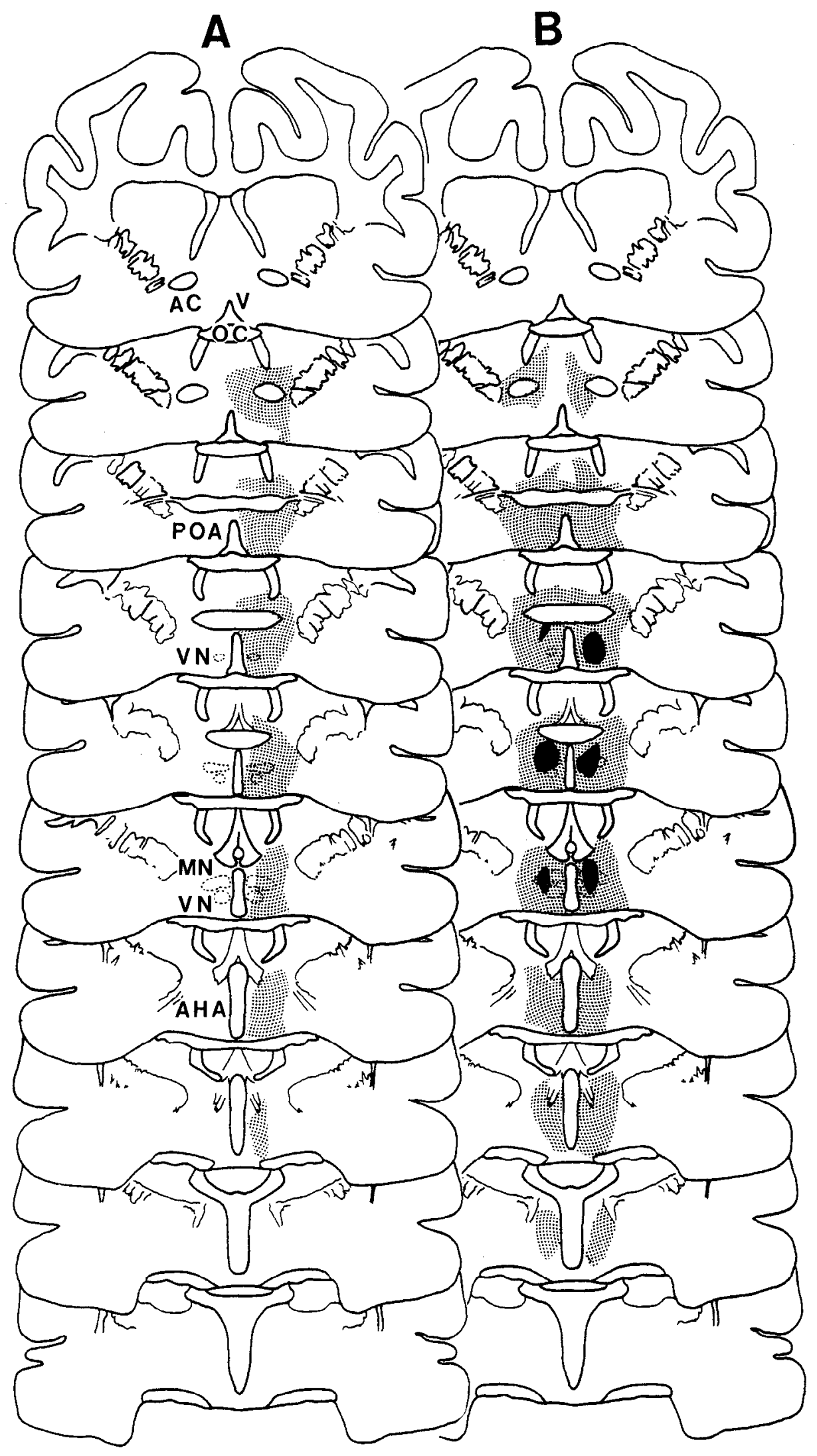

Figure 3. Composite diagrams showing the extent of excitotoxic damage caused by infusions of quinolinic acid into the dorsomedial preoptic area/anterior hypothalamus (mPOA/AH) of groups of ferrets from Experiment 2. Hatched regions depict the maximal extent of tissue which sustained damage in any member of a particular group; black regions depict overlap of excitotoxic damage for all members of a particular group. Data are shown for 7 males with unilateral excitotoxic damage ( 3 and 4 males with damage in the left and right sides, respectivelysummarized here on one side for ease of presentation) $(A)$ and for 12 males with bilateral excitotoxic damage $(B)$ of the mPOA/AHA. Abbreviations: $P O A$, preoptic area; $v n$, nondimorphic, ventral nucleus of the mPOA/AH; $m n$, sexually dimorphic male nucleus of the mPOA/AH; $A H A$, anterior hypothalamic area. 


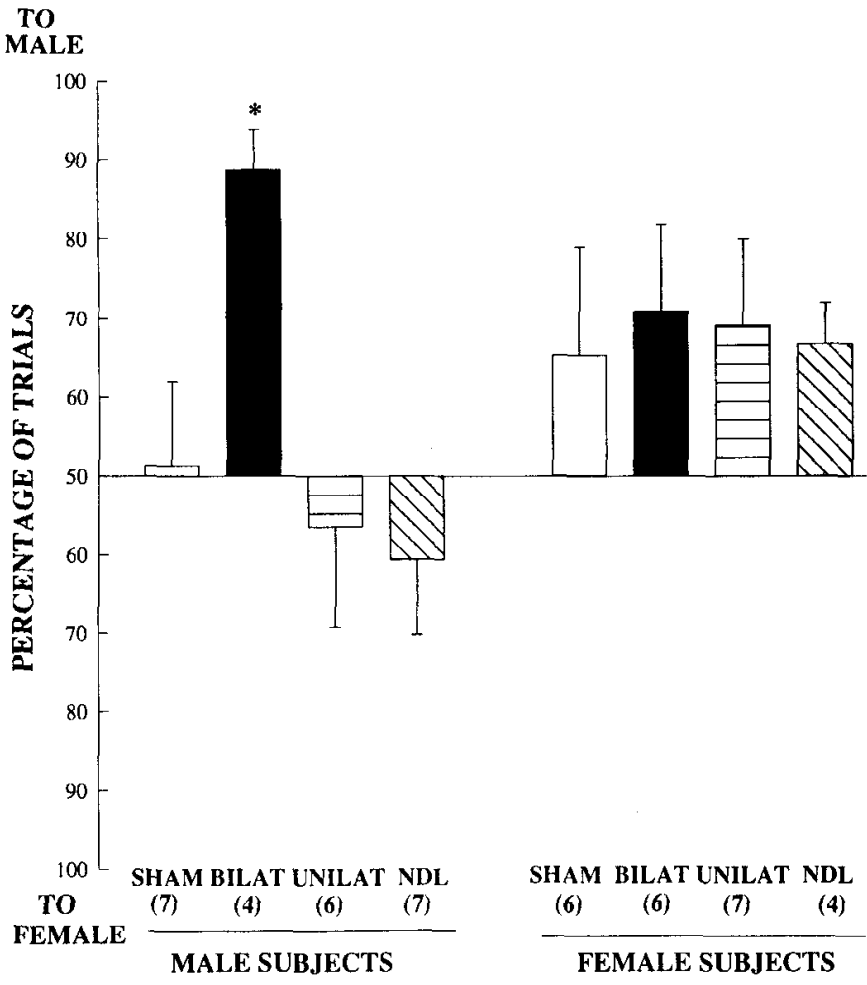

Figure 4. Percentage of free trials in T-maze tests of partner preference during which gonadectomized, estrogen-treated subjects in Experiment 1 chose to approach either a sexually active stimulus male (to malc) or a sexually receptive stimulus female (to female). $\Lambda$ bbreviations: $S H A M$, bilateral infusions of PBS vehicle into the dorsomedial preoptic area/anterior hypthalamus (mPOA/AH); BILAT, bilateral; $U N-$ $I L A T$, unilateral excitotoxic lesions of the InPOA/AH. $N D L$, no detectible excitotoxic damage found, despite previous bilateral infusions of quinolinic acid into the mPOA/AH. ${ }^{*}$, Significantly different $(p<0.05)$ from sham, unilat and NDL males by post-hoc two-tailed Mann-Whitney $U$ tests. Additional comparisons showed that values for males with unilateral mPOA/AH lesions differed significantly $(p<0.05)$ from those of females bearing bilateral, unilateral or no detectible damage while values for males with no detectible lesion differed significantly $(p<0.05)$ from all groups of females. Data are expressed as mean + SEM; number of subjects/group is given in parentheses.

An analysis of the behaviors that occurred between subjects and the stimulus male during the 1 min interactions which followed each trial (free and guided) to the stud male revealed no differences between males bearing bilateral mPOA/AH lesions and males in the three control groups for each experiment. Male subjects were more likely than females to establish neck grips on a stimulus male. However, the incidence of this behavior was very low in all male groups, and it seems unlikely that the opportunity to engage in this behavior provided the added incentive for males bearing mPOA/AH lesions to approach the stud male. The same argument can be made for the opportunity to engage in aggressive behavior with the stud, in so far as very little fighting was observed in either experiment and when it did occur, there were no differences among groups of males in its incidence. In both experiments males were less likely than females to receive neck grips from the stimulus male. However, when stimulus males did establish a neck grip, male subjects in all groups were as likely as females to respond with a receptive posture. This observation agrees with previous results (Baum et al., 1985) showing that receptive coital capacity is not sexually differentiated in the ferret. However, males' uniform willingness

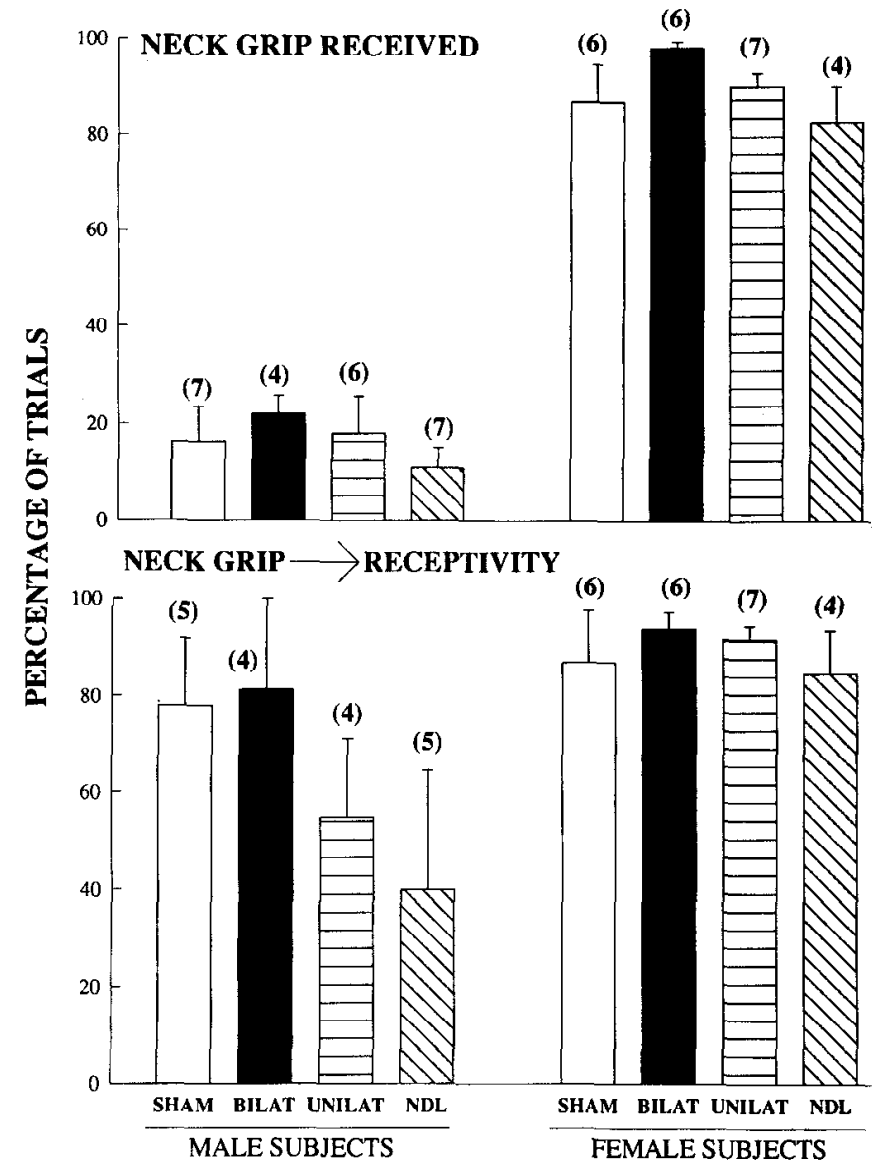

Figure 5. Percentage of trials (free + guided) when subjects in different groups from Experiment 1 approached a stimulus male and received a neck grip (top panel) or in which these subjects displayed a receptive response after receipt of a neck grip from the stimulus male (bottom panel). See caption to Figure 4 for group definitions. Data are expressed as mean \pm SEM; the number of subjects/group is given in parentheses.

to display receptive behavior in response to neck gripping by a stud cannot explain the significantly greater preference of males with mPOA/AH lesions to approach a stimulus male. More research is needed to identify the rewarding features of the stud malc which underlie the obscrved lesion-induced increments in males' preference to approach them.

In both experiments EB-treated males with bilateral mPOA/ AH lesions were significantly less likely than control males to establish a neck grip on the estrous female in trials when they chose this stimulus in the T-maze. In Experiment 2 gonadectomized ferrets were also tested for masculine coital behavior while receiving a high dosage of TP. Males with bilateral mPOA/ $\mathrm{AH}$ lesions displayed significantly lower levels of neck grip and mounting behavior than control males. These findings corroborate numerous previous studies (reviewed in Sachs and Meisel, 1988) showing that lesions of the mPOA $/ A H$ disrupt masculine coital performance in male vertebrates. Our findings also raise the possibility that male ferrets with bilateral mPOA/AH lesions stopped approaching stimulus females in the $\mathrm{T}$-maze because they derived less reward from coital interactions with these animals. Everitt (Everitt and Stacey, 1987; Hughes et al., 1990) has put forward such an argument to explain the gradual deterioration in the operant performance to gain access to an estrous female, or to stimuli previously associated with an estrous fe- 


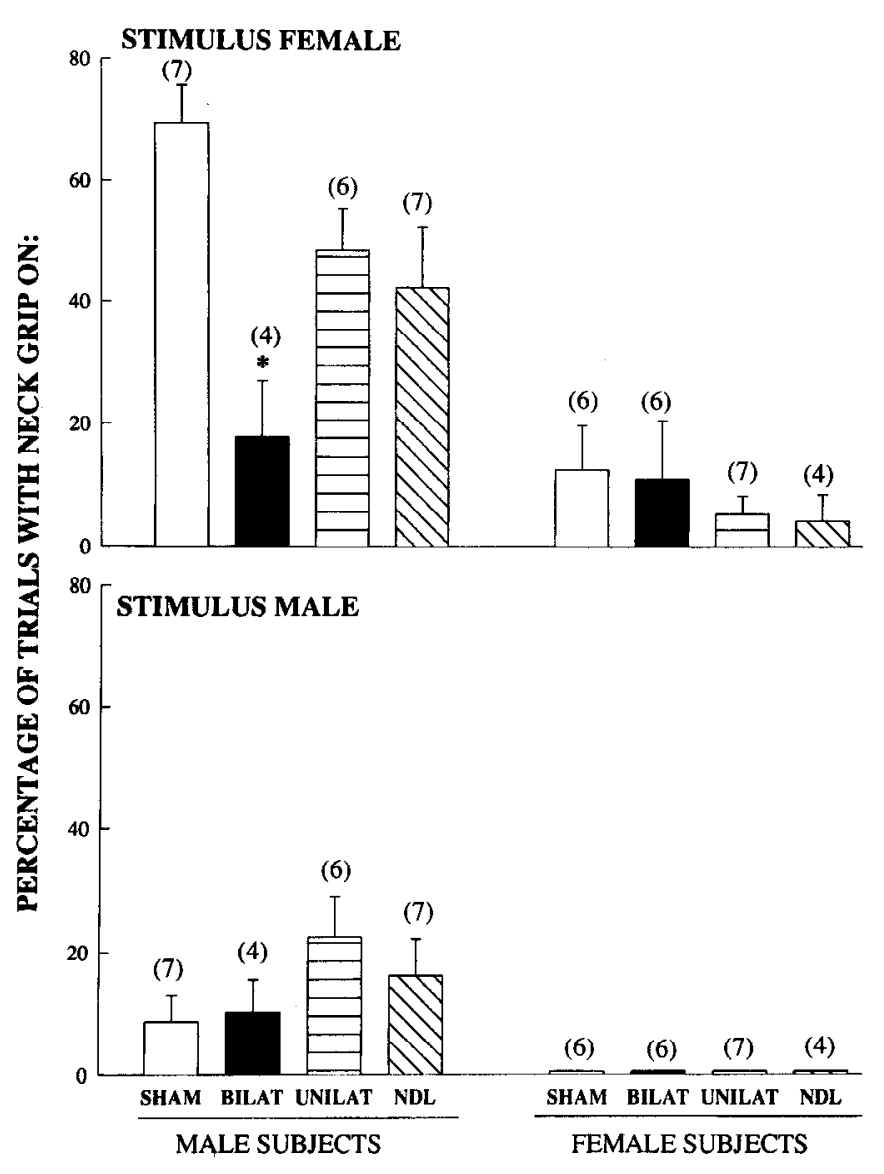

Figure 6. Percentage of trials (free + guided) during which subjects in different groups from Experiment 1 performed a neck grip on either a stimulus female (top panel) or a stimulus male (bottom panel). See caption to Figure 4 for group definitions. *, Significantly different ( $p$ $<0.05$ ) from other groups of males by post-hoc two-tailed Mann-Whitney $U$ tests. Data are expressed as mean \pm SEM; the number of subjects/group is given in parentheses.

male, of male rats after bilateral mPOA/AH lesions. In Experiment 1 , male subjects had received only a minimal exposure to estrous females (in guided trials to this stimulus) at the time they were expressing a preference to approach the stimulus male in T-Inaze lests given after the administration of bilateral $\mathrm{mPOA} /$ $\mathrm{AH}$ lesions. In Experiment 2, males had been tested extensively with a receptive female following the placement of bilateral $\mathrm{mPOA} / \mathrm{AH}$ lesions, and prior to the administration of partner preference tests in the T-maze. Thus, we cannot rule out a reduction in reward derived from the female, resulting from deficient mating performance, as a factor contributing to the altered preference pattern displayed by these lesioned males. It is worth noting, however, that bilateral $\mathrm{mPOA} / \mathrm{AH}$ lesions attenuated the duration of neck grip and mounting behavior in male ferrets, but by no means eliminated these behaviors during two 20 min tests (see Fig. 8).

It can be argued that the observed reduction in neck grip and mounting behavior displayed toward stimulus females by males bearing bilateral $\mathrm{mPOA} / \mathrm{AH}$ lesions was a symptom, as opposed to a cause, of a lesion-induced disruption of masculine psychosexual motivation. This view is supported by results of two studies, one using rats (Paredes et al., 1993) and another using marmosets (Lloyd and Dixson, 1988), in which lesions of the mPOA/AH significantly reduced aspects of males' precopulatory

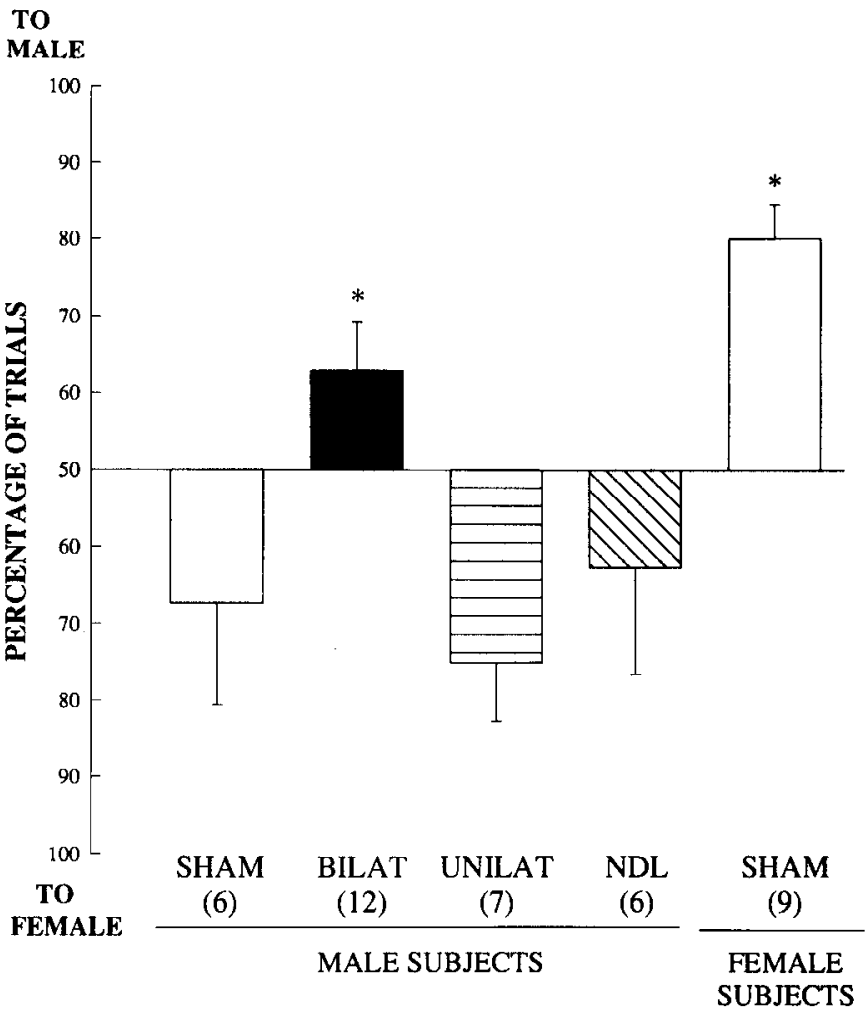

Figure 7. Percentage of free trials in T-maze tests of partner preference during which gonadectomized, estrogen-treated subjects in Experiment 2 chose to approach either a sexually active stimulus male (to male) or a sexually receptive stimulus female (to female). See caption to Figure 4 for group definitions. *, Significantly different $(p<0.05)$ from sham, unilat, and NDL males by post-hoc two-tailed Mann-Whitney $U$ tests. Values for males with bilateral mPOA/AH lesions were also significantly $(p<0.05)$ lower than values for sham-operated female subjects. Data are expressed as mean \pm SEM; number of subjects/ group is given in parentheses.

behavior. Likewise, male rats' preference to approach and interact with a tethered estrous, as opposed to an anestrous female, was significantly attenuated by mPOA/AH lesions (Edwards and Einhorn, 1986) although bilateral sagital knife cuts which isolated the mPOA/AH failed to attenuate males' preference for odors derived from an estrous female (Szechtman et al., 1978). Reports (Everitt and Stacey, 1987; Hughes et al., 1990) that male rats with $\mathrm{mPOA} / \mathrm{AH}$ lesions persisted in performing operant responses to gain access to an estrous female or chose to remain in an environment previously associated with mating until they had experienced the frustration of being unable to copulate with an estrous female are not necessarily incompatible with this view. In both cases the persistent operant performance depended on secondary reinforcers previously associated with mating. In experiments (such as the present ones) in which males were confronted directly with stimulus females following the placement of lesions in the mPOA/AH, deficits in approach as well as precopulatory behaviors were immediately apparent. Finally, it is difficult to see how a reduction in the capacity of males with bilateral $\mathrm{mPOA} / \mathrm{AH}$ lesions to execute coital behaviors with an estrous female can explain the preference of these males to choose to approach and intcract with a stimulus male during T-maze tests. On any trial (free or guided) subjects could have chosen to remain in the start area instead of approaching either of the stimulus animals. In fact, this almost never happened. 


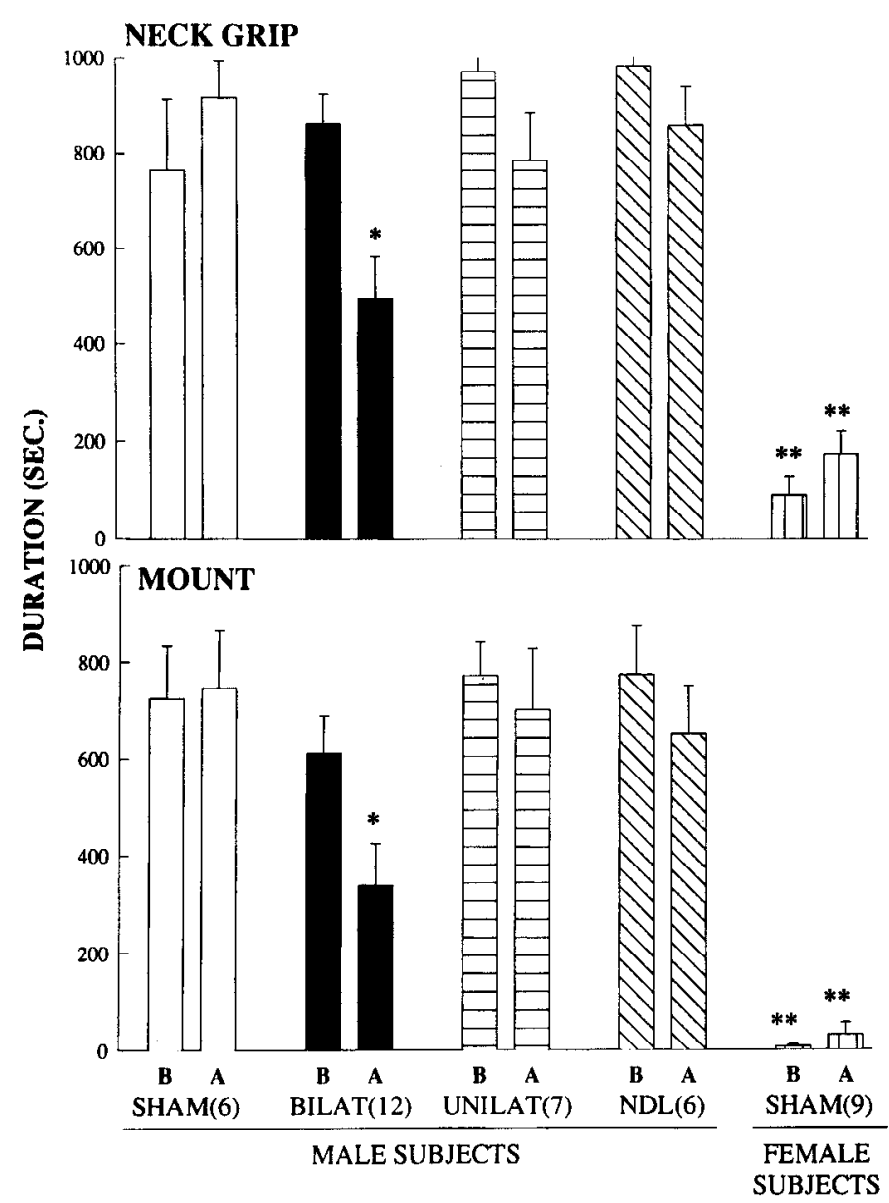

Figure 8. Masculine coital behaviors displayed by different groups of gonadectomized, testosterone-propionate-treated male and female ferrets in tests with an estrous female given before $(B)$ and after $(A)$ brain surgery. See caption to Figure 4 for group definitions. *, Significantly different $(p<0.05)$ from preoperative values; **, significantly different from preopcrative and postoperative Sham-operated male values by post-hoc Newman-Keuls tests. Data are expressed as mean \pm SEM; number of subjects/group is given in parentheses.

Males with bilateral mPOA/AH lesions, like all groups of females, were more likely than control males to approach and interact with the stud male.

Another possible explanation for the lesioned-induced change in males' partner preference is that characteristics of the estrous female became aversive to males after brain surgery was performed. There is, however, little support for this view from the data collected. In both experiments the latency to approach the estrous female, both in free and guided trials, was similar among all groups. Also, in a pilot study (data not shown) a subset of males from the various groups included in Experiment 2 received simultaneous access to bowls containing feces and urine collected from a stimulus male and female. All males, including those bearing bilateral mPOA/AH lesions, spent twice as much time investigating the female excretia. This observation corroborates a previous study (Clapperton et al., 1988) showing that male ferrets preferred to approach anal sac odors derived from females as opposed to males. Thus, there is no indication that males bearing bilateral mPOA/AH lesions found females' chemosensory or behavioral attributes to be aversive.

Previous studies have not rigorously assessed subjects' preference for male versus female sexual partners after the place- ment of mPOA/AH lesions. In several instances, however, males' proceptive and receptive responsiveness to a sexually active stimulus male have been studied. If anything, this behavior was enhanced following bilateral lesions of the mPOA/AH. Thus, in ferrets large bilateral electrolytic lesions of the POA/ $\mathrm{AH}$ or more restricted bilateral damage to the sexually dimorphic Mn-POA/AH caused males to run progressively faster (in an L-maze) to a stud male in response to increasing doses of $\mathrm{EB}$, a response characteristic of control females (Cherry and Baum, 1990). In rhesus monkeys (Slimp et al., 1978) bilateral radiofrequency lesions centered in the dorsomedial POA/AH caused striking deficits in masculine coital behavior in tests with sexually active females. Lesioned males also showed increased numbers of presentations after receiving sex contacts from stimulus females. Unfortunately, no attempt was made in that study to assess the proceptive or receptive sexual behavior of lesioned males in tests with a sexually active stimulus male. In the cat (Hart and Leedy, 1983) bilateral radiofrequency lesions of the mPOA/AH augmented males' proceptive and receptive responsiveness to a stimulus male. Similar results have been obtained in male rats (Hennesey et al., 1986; Kondo et al., 1990; Olster, 1993) and guinea pigs (Rodriguez-Sierra and Terasawa, 1979). Taken together, the results of all of these studies would have predicted the outcome of the present experiments, namely, that when given a choice, males with bilateral lesions of the mPOA/ AH prefer to approach and interact sexually with an active male as opposed to a receptive female. This result implies that features of mPOA/AH structure are sexually differentiated in males and contribute in an essential way to heterosexual partner preference. We cannot say with certainty whether neurons of the sexually dimorphic Mn-POA/AH, present only in male ferrets, are responsible for males' preference to approach and interact with an estrous female. In Experiment 2 efforts to restrict excitotoxic damage to this nuclcus werc not successful, although in both Experiments 1 and 2 the portion of the dorsomedial $\mathrm{POA} / \mathrm{AH}$ which was damaged in all bilaterally lesioned subjects included this region. More research is needed to explore this question.

In the present experiments the effect of excitotoxic mPOA/ AH lesions on males' partner preference was assessed after gonadectomy and prolonged treatment with a high dose of estrogen. Gonadally intact males, even when they are in breeding condition, would never experience this degree of estrogenic stimulation in the absence of concurrent androgen receptor activation. We administered EB in these experiments because in a previous study (Stockman et al., 1985) we had seen a more robust sex dimorphism in sexual partner preference when gonadectomized ferrets were treated with estrogen instead of testosterone. Thus we reasoned that any possible effect of mPOA/AH lesions on males' partner preference would best be revealed under circumstances in which a sex difference in this behavior is also present. In fact, we did observe a consistent preference for estrogenprimed males with mPOA/AH lesions to approach a stud male. We do not know whether this preference would be expressed in lesioned males which are tested while receiving $\mathrm{T}$.

The absence of any effect of bilateral $\mathrm{mPOA} / \mathrm{AH}$ lesions on females' preference to approach and interact with a stimulus male (Experiment 1) differs from results of several previous studies using rats, in which either radio-frequency (Whitney, 1986) or excitotoxic (Hoshina et al., 1994) lesions of the mPOA/ AH significantly reduced proceptive and soliciational behaviors directed toward stimulus males. Our findings also seem to differ 
from results of two studies using female marmosets, in which radio-frequency (Kendrick and Dixson, 1986) and excitotoxic lesions of the anterior-medial hypothalamus (Dixson and Hastings, 1992) reduced the display of proceptive responses. It should be noted, however, that none of these studies employed an operant assessment of females' motivational state, such as the one used in the present experiments. Also, the female ferret displays no obvious appetitive, (proceptive) responses such as hopping, darting, and ear-wiggling (rats) or tongue-flicking (marmosets) which might otherwise have been disrupted by mPOA $/$ AH lesions.

There are several reports for humans (reviewed in Tobet and Fox, 1992) that the volume of subnuclei within the POA/AH continuum is larger in males than in females. Thus, Allen et al. (1989) and LeVay (1991) reported that the third interstitial nucleus of the anterior hypothalamus (INAH-3) is sexually dimorphic (larger in men than in women), although prior to this Swaab and Fliers (1985) had reported a sex dimorphism in anuther nucleus, identified as INAH-1 by Allen et al. (1989). In addition, I.eVay (1991) reported that the volume of INAH-3 in the brains of homosexual men was significantly lower than in heterosexual men, and thus resembled the volume of INAH-3 in heterosexual women. From this correlational study there is no way of knowing whether the reported differences in INAH-3 volume, either between heterosexual men and women or between heterosexual and homosexual men, contributes in anyway to the respective differences between these groups in sexual partner preference. Results of the present experiments using ferrets, in which the effect mPOA/AH neuronal destruction on sexual partner preference as well as masculine sexual behavior were rigorously assessed, support the possibility that groups of neurons in the human PUA/AH continuum somehow influence sexual orientation as well as masculine coital function. The limitation of the ferret model is quickly apparent, however, in so far as the increased preference for the male partner seen in males given bilateral mPOA/AH lesions was associated with significant reductions in masculine coital performance. The available data suggest that coital function in homosexual men is no different from that of heterosexual men (see Baum et al., 1990a, and Breedlove, 1992, 1994, for further discussions of the limitations of animal Inodels for sludying neuroendocrine factors in human sexual orientation).

\section{References}

Adkins-Regan E, Ascenzi M (1987) Social and sexual behaviour of male and female zebra finches treated with oestradiol during the nestling period. Anim Behav 35:1100-1112.

Allen LS, Hines M, Shryne JE, Gorski RA (1989) Two sexually dimorphic cell groups in the human brain. J Neurosci 9:497-506.

Bakker J, Brand T, van Ophemert J, Slob AK (1993) Hormonal regulation of adult partner preference behavior in neonatally ATD-treated male rats. Behav Neurosci 107:480-487.

Baum MJ (1976) Effects of testosterone propionate administered perinatally on sexual behavior of female ferrets. J Comp Physiol Psychol 90:399-410.

Baum MJ, Everitt BJ (1992) Increased expression of c-fos in the medial preoptic area after mating in male rats: role of afferent inputs from the medial amygdala and midbrain central tegmental field. Neuroscience 50:627-646.

Baum MJ, Stockman ER, Lundell LA (1985) Evidence of proceptive without receptive defeminization in male ferrets. Behav Neurosci 99: 742-750.

Baum MJ, Carroll RS, Cherry JA, Tobet SA (1990a) Steroidal control of behavioural, neuroendocrine and brain sexual differentiation: studies in a carnivore, the ferret. J Neuroendocrinol 2:401-418.

Baum MJ, Erskine MS, Kornberg E, Weaver CE (1990b) Prenatal and neonatal testosterone exposure interact to affect differentiation of sexual behavior and partner preference in female ferrets. Behav Neurosci 104:183-198.

Beach FA (1976) Sexual attractivity, proceptivity and receptivity in female mammals. Horm Behav 7:105-138.

Breedlove MS (1992) Sexual dimorphism in the vertebrate nervous system. J Neurosci 12:4133-4142.

Breedlove MS (1994) Sexual differentiation of the human nervous system. Annu Rev Psychol 45:389-418.

Carroll RS, Weaver CE, Baum MJ (1988) Evidence implicating aromatization of testosterone in the regulation of male ferret sexual behavior. Physiol Behav 42:457-460.

Cherry JA, Baum MJ (1990) Effects of lesions of a sexually dimorphic nucleus in the preoptic/anterior hypothalamic area on the expression of androgen and estrogen-dependent sexual behavior in male ferrets. Brain Res 522:191 203.

Clapperton BK, Minot EO, Crump DR (1988) An olfactory recognition system in the ferret Mustela Furo L. (Carnivora:Mustelidae). Anim Behav 36:541-553.

Commins D, Yahr, P (1984) Adult testosterone levels influence the morphology of a sexually dimorphic area in the Mongolian gerbil brain. J Comp Neurol 224:132-140.

Dixson AF, Hastings MH (1992) Effects of ibotenic acid-induced neuronal degeneration in the hypothalamus upon proceptivity and sexual receptivity in the female marmoset (Callithrix jacchus). J Neuroendocrinol 4:719-726.

Dohler KD, Srivastava SS, Shryne JE, Jarzab B, Sipos A, Gorski RA (1984) Differentiation of the sexually dimorphic nucleus in the preoptic area of the rat brain is inhibited by postnatal treatment with an estrogen antagonist. Neuroendocrinology 38:297-301.

Edwards DA, Einhorn LC (1986) Preoptic and midbrain control of sexual motivation. Physiol Behav 37:329-335.

Everitt BJ, Stacey P (1987) Studies of instrumental behavior with sexual reinforcement in male rats (Rattus norvegicus). II. Effects of preoptic area lesions, castration and testosterone. J Comp Psychol 101:407-419.

Ford JJ (1983) Postnatal differentiation of sexual preference in male pigs. Horm Behav 17:152-162.

Gorski RA, Gordon JH, Shryne JE, Southam AM (1978) Evidence for a morphological sex difference within the medial preoptic area of the rat brain. Brain Res 148:333-346.

Hart BL, Leedy MG (1983) Female sexual responses in male cats facilitated by olfactory bulbectomy and medial preoptic/anterior hypothalamic lesions. Behav Neurosci 4:608-614.

Hennessey AC, Wallen K, Edwards DA (1986) Preoptic lesions increase the display of lordosis by male rats. Brain Res 370:21-28.

Hoshina Y, Takeo T, Nakano K, Sato T, Sakuma Y (1994) Axon-sparing lesions of the preoptic area enhance receptivity and diminishe proceptivity among components of female rat sexual hehavior. Behav Brain Res 61:197-204.

Hughes AM, Everitt BJ, Herbert J (1990) Comparative effects of preoptic area infusions of opioid peptides, lesions and castration on sexual behaviour in male rats: studies of instrument behaviour, conditioned place preference and partner preference. Psychopharmacology 102:243-256.

Johnson WA, Tiefer L (1972) Sexual preferences in neonatally castrated male golden hamsters. Physiol Behav 9:213-218.

Kendrick KM, Dixson AF (1986) Anteromedial hypothalamic lesions block proceptivity but not receptivity in the female common marmoset. Brain Res 375:221-229.

Kondo Y, Shinoda A, Yamanouchi K, Arau Y (1990) Role of septum and preoptic area in regulating masculine and feminine sexual behavior in male rats. Horm Behav 24:421-434.

Lambert GM, Baum MJ (1991) Reciprocal relationships between pulsatile androgen secretion and the expression of mating behavior in adult male ferrets. Horm Behav 25:382-393.

LeVay S (1991) A difference in hypothalamic structure between heterosexual and homosexual men. Science 253:1034-1037.

Lloyd SAC, Dixson AF (1988) Effects of hypothalamic lesions upon the sexual and social behavior of the male common marmoset. Brain Res 463:317-329.

Olster DH (1993) Ibotenic acid-induced lesions of the medial preoptic area/anterior hypothalamus enhance the display of progesterone-facilitated lordosis in male rats. Rrain Res 626:99-105.

Paredes RG, Highland L, Karam P (1993) Socio-sexual behavior in 
male rats after lesions of the medial preoptic area: evidence for reduced sexual motivation. Brain Res 618:271-276.

Rodriguez-Sierra JF, Teresawa E (1979) Lesions of the preoptic area facilitate lordosis behavior in male and female guinea pigs. Brain Res Bull 4:513-517.

Sachs BD, Meisel R (1988) The physiology of male sexual behavior In: The physiology of reproduction (Knobil E, Neil J, eds), pp 13931485. New York: Raven.

Slimp JC, Hart BL, Goy RW (1978) Heterosexual, autosexual and social behavior of adult male rhesus monkeys with medial preopticanterior hypothalamic lesions. Brain Res 142:105-122.

Stockman ER, Callaghan RS, Baum MJ (1985) Effects of neonatal castration and testosterone treatment on sexual partner preference in the ferret. Physiol Behav 34:409-414.

Swabb DF, Fliers E (1985) A sexually dimorphic nucleus in the human brain. Science 228:1112-1115.

Szechtman H, Caggiula AR, Wulkan D (1978) Preoptic knife cuts and sexual behavior in male rats. Brain Res 150:569-591.
Tobet SA, Fox TO (1992) Sex differences in neuronal morphology influenced hormonally throughout life. In: Handbook of bchavioral neurobiology, Vol 11, Sexual differentiation (Gerall AA, Moltz H, Ward IL, eds), pp 41-83. New York: Plenum.

Tobet SA, Zahniser DJ, Baum MJ (1986a) Sexual dimorphism in the preoptic/anterior hypothalamic area of ferrets: effects of adult exposure to sex steroids. Brain Res 364:249-257.

Tobet SA, Zahniser DJ, Baum MJ (1986b) Differentiation in male ferrets of a sexually dimorphic nucleus of the preoptic/anterior hypothalamic area requires prenatal estrogen. Neuroendocrinology 44: 299-308.

Whitney JF (1986) Effect of medial preoptic lesions on sexual behavior of female rats is determined by test situation. Behav Neurosci 100: 230-235.

Yahr P, Gregory JE (1993) The medial and lateral cell groups of the sexually dimorphic area of the gerbil hypothalamus are essential for male sex behavior and act via separate pathways. Brain Res 631: 287-296. 